\title{
Presidential Address 2018: Towards professional excellence in sexual health and HIV care
}

\author{
Chief Guest, Dr Kulasiri Buddhakorala, \\ Guest of Honour, Professor Graham Taylor, \\ Distinguished overseas guests, Representatives from \\ UN agencies, and International Partner organiza- \\ tions,
}

Past presidents, Founder members, Fellows, my office bearers and council members and all other members of the College,

Distinguished invitees from Ministry of health,

My dear teachers, colleagues, staff members, ladies and gentlemen,

Good evening, it is indeed one of the happiest days of my life. It is a great pleasure and honour to address you as the president of Sri Lanka College of Sexual Health and HIV medicine this evening.

The theme of this 23rd annual scientific sessions of Sri Lanka college of sexual health and HIV medicine and my presidential speech is "Towards professional excellence in sexual health and HIV care" with emphasis to Sri Lankan health system.

Before I speak on professional excellence in sexual health and HIV care, let me begin by digging into the history of Sri Lanka to find and understand the foundation and evolution of our medical system.

History of disease should be old as the history of mankind. Recorded history of Sri Lanka runs back to $6^{\text {th }}$ century BC when Vijaya and his team arrived from North India. Later, Buddhism was introduced to King Devanampiyathissa in the $3^{\text {rd }}$ century BC by Arhat Mihindu and his group of Buddhist monks at a mountain peak named Mihintale, closer to Anuradhapura. Why I specially mentioned these two events from the history; because these two events would have contributed a lot to the history of medicine in Sri Lanka.

Buddhism commend, care of sick as a meritorious act of highest order which would have encouraged kings to improve medical services in the country. Mihintale Hospital, which was built in the 9th century AD or much earlier (Attributed to King Sena II) has been described as the oldest hospital in the world. But, beyond this history, some believe of a pre-historic king of Lanka named "Ravana" as a great physician who had written medical books namely, "Kumara Thanthraya" and Arka Prakashaya" etc. Additionally, we would have had charms and incantations as methods of healing. The earliest system of medicine existed in Ceylon is known as "Deshiya Chikitsa" or "Sinhala Vedakama" which was handed down from generation to generation. Later this was integrated with the Ayurveda system and was continued as the main medical system of the country through Portuguese and Dutch colonial era. During the British era, popularity of Ayurveda began to decline with the loss of royal patronage and the introduction of western medical system to the country by the British administration.

Looking back into the history of sexual health in Sri Lanka, an ancient medical book named "Saaraartha Sangrahaya", (Ruwangiri Rajamaha Viharaya, Debalgala, Kahatagasdigiliya) composed by King Buddhadasa during $5^{\text {th }}$ Century AD describes a venereal disease similar to syphilis.

Later in 1500-1600 AD, a disease named "Parangi" was considered as a highly contagious disease (Later identified as Yaws) in Ceylon, but most likely, syphilis was also prevalent among Portuguese during that time. (Ariyaratna de Silva and Michael G Gomez-Genitourin Med,1994;70:349-354).

The Contagious Diseases Ordinance, enacted in 1867, sought to limit the consequences of venereal disease by the registration and periodical examination of women openly carrying on prostitution. (Eunice D. C. Pereira and C. S. Ratnatunga, Brit. J. Vener. Dis. (1965), 41, 97).

The venereal disease commission of Ceylon was formed in 1921, and a clinic was established in the Port of Colombo. In 1939 late Dr H. C. P. Gunewardene, who was specially trained in venereal disease at the Whitechapel Clinic, London, returned to the island and was appointed Surgeon-in-Charge of Genito-urinary Clinic, Colombo; this being the new designation given to the old VD Clinic.

Much organized "Anti Venereal Disease (VD) Campaign" was formed in 1952 with the support of WHO-Geneva with the following objectives:

To establish a model venereal disease clinic in Colombo which would serve as, the chief clinic of Ceylon, and the training centre for medical and para-medical staff. 
Develop a full venereal disease service with trained staff in the major outstations.

Develop the diagnostic and treatment facilities for seafarers in the Port of Colombo.

Train local staff in simple serological testing so as to provide such facilities in the main outstations.

Most importantly, to establish serological testing of expectant mothers as a routine and thus control congenital syphilis.

Ladies and Gentlemen, let me turn the page from past to present.

These historical initiations, interventions and commitments, provided us a solid platform to reach what we are today.

At the beginning, our services were limited to prostitutes, seafarers and expectant mothers, but gradually the scope of preventive and curative services provided by us were extended to more key populations such as Drug Users, Men having Sex with Men (MSM), Beach Boys and also to the general population.

Meanwhile, various terminologies used in the sector were transformed from venereal diseases or VD to sexually transmitted diseases (STDs), Sexually Transmitted infections (STIs) including genital dermatoses and finally to represent overall sexual health and HIV medicine.

The National STD/AIDS Control Programme of Sri Lanka is reinforced with well-established country policies, strategic plans, guidelines etc. There is a well-trained dedicated healthcare work force and a strong service delivery system covering the entire island. The campaign is an entity that is vertically driven and independently financed through the budgetary mechanism of "Government of Sri Lanka". However, we are fortunate to receive financial support from other global financing organizations. Furthermore, the campaign is equipped with wellorganized strategic information and monitoring system that enables us to manage our data efficiently and effectively.

We were able to maintain very low prevalence of STIs including HIV among the general population and low prevalence even among key populations. We have reached the elimination of mother to child transmission of HIV and syphilis and we expect WHO certification in early 2019.
Ministry of health and NSACP conduct pre-service and in-service training programmes for doctors and other staff. PGIM with Board of Study in Venereology have designed a post graduate diploma and MD in venereology to produce specialists and consultants in the field of venereology. There are more than 40 venereologists who serve locally and in overseas countries such as United Kingdom and Australia and similar number of postgraduate trainees are being trained to strengthen the specialist services in the future. There are more than 30 STD clinics and several branch clinics under the umbrella of National STD/AIDS Control Programme, most headed by consultants with a trained team of medical officers and paramedical staff to provide services all around the country. Using these resources, we are targeting to reach UNAIDS $90-90-90$ targets by 2020 to end AIDS by 2025 in Sri Lanka, expectantly 5 years ahead of the global target.

Ladies and Gentlemen,

Sri Lanka College of Sexual Health and HIV medicine; formerly known as Sri Lanka College of Venereologists, and National STD/AIDS Control Programme of Sri Lanka have been promoting professionalism among members and employees, may be not with modern terminology or definitions but with their own ways of mentoring and teaching. While being happy and proud for what has been achieved to date, it is high time that the council looked for the best quality; i.e. target to achieve "Professional excellence in sexual health and HIV care"!

I will try to express my views on this aspect over next half of my presentation.

Most of us have taken an oath prior to practicing as a medical doctor. In Sri Lanka, still it is the Hippocratic Oath, which is the foundation of professionalism and medical ethics known in western medical history. Other countries which practice western medicine still use newer versions of the Hippocratic Oath. I still remember, and may be most of you; the day we took that oath, the day that a responsible doctor entered into us. Should it be a one-off thing? My answer is "No". Some countries have developed new "codes of conduct" for medical practitioners to fill the gap, to face problems of today's changing health care climate.

"Good Medical Practice for Physicians" is one such guidance developed by the British General Medical Council to maintain these qualities of professionalism among their membership. I believe 
this has contributed to raise standards and quality in terms of professionalism of physicians in the UK. Some other countries, for example, the USA and Australia have similar national or regional documents for the same intention.

I thought of sharing some important qualities extracted from them that we could incorporate in to our system in view of achieving our target.

\section{Clinical Governance and sexual health}

UK Department of Health defines clinical governance as "the framework through which organisations are accountable for continuously improving the quality of their services and safeguarding high standards of care by creating an environment in which excellence in healthcare will flourish".

Principles of clinical governance could be used to raise the standards of care in sexual health and HIV.

The first element is, "Patient safety". This includes; Incident Management, Risk Management, Alerting System, Waste Management, Medicines Optimisation, Safe Environment and Safeguarding.

The second Element is "Clinical Effectiveness". This includes; Cost effectiveness, Clinical Guidelines, Evidence-based practice, Care pathways, Clinical Audits, Policy Development, Claims Management, Information Governance, Staff Management, Education and Training and Equality and Diversity.

The third element is "Patient and Public Experience". This includes; Complaints Management, Consent, Nutrition and Hydration, Patient and Public Information, Patient and Public Involvement and their needs.

How can we achieve professional excellence in "Patient Safety and quality"?

We must contribute to and comply with systems to protect patients by,

Regular reviews and audits.

Responding constructively to their outcomes.

Report suspected adverse drug reactions.

Reviewing patient feedback where it is available.

Adverse event recognition.
We must respond to risks to safety by,

Creating a culture that allows all staff to raise concerns openly and safely.

Vulnerable adults or children and young people as patients, we should consider their needs and welfare and offer them help if we think their rights have been abused or denied.

Also, risks posed by your health to be considered.

How to achieve excellence in knowledge, skills and performance?

Develop and maintain professional performance,

We must be competent in all aspects of our work, including management, research and teaching.

We must keep our professional knowledge and skills up to date. This requires some self-reflection to assess where improvement is needed.

We must regularly take part in activities that maintain and develop our competence and performance.

We must be familiar with guidelines and developments that affect our work and keep up to date.

We must follow the law and other regulations relevant to our work.

We must take steps to monitor and improve the quality of our work. This improvement required is not limited to individual level, but of the system and public health as well. For example, improvement of laboratory facilities, testing and stock management, improving antiretroviral therapy regimens and maintain uninterrupted supply, improving infection control, safety measures etc .

Apply knowledge and experience to practice,

We must recognise and work within the limits of our competence.

We must adequately assess the patient's conditions, taking in to account of their history, their views and values as well.

We may have to refer a patient to another practitioner when this serves the patient's needs.

We must have consent or other valid authority before we carry out any examination or investigation, provide treatment or involve patients 
or volunteers in teaching or research.

We must record our work clearly, accurately and legibly and should make records at the same time as the events you are recording or as soon as possible, afterwards.

We must keep records that contain personal information about patients, colleagues or others securely.

Communication, partnership and teamwork are other very important aspects in professionalism.

We must listen to patients, take account of their views, and respond honestly to their questions.

We must give patients the information they want or need to know in a way they can understand.

We must be considerate to those close to the patient and be sensitive and responsive in giving them information and support.

When we are on duty we must be readily accessible to patients and colleagues seeking information, advice or support.

It is important to establish and maintain partnerships with patients. To excel in this,

We must be polite and considerate, treat patients as individuals and respect their dignity and privacy.

We must treat patients fairly and with respect whatever their life choices and beliefs.

We must work in partnership with patients, sharing with them the information they will need to make decisions about their care including: their condition, its likely progression and the options for treatment, including associated risks and uncertainties, the progress of their care, and our role and responsibilities in the team, who is responsible for each aspect of patient care, and how information is shared within teams and among those who will be providing their care any other information patients need if they are asked to agree to be involved in teaching or research.

We must treat information about patients as confidential and this includes after a patient has died.

We must support patients in caring for themselves, to empower them to improve and maintain their health. This may, for example, include: advising patients on the effects of their life choices and lifestyle on their health and well-being supporting patients to make lifestyle changes where appropriate. In providing this information you must not imply or express disapproval of the patient's lifestyle choices or beliefs.

Accountability is another aspect of professionalism that requires the physician to avoid letting selfinterest override the patients' interest.

This includes ownership of patient outcomes and follow-up. Physicians are often viewed as leaders both inside and outside of health care.

Leadership defines culture! And if we want to create a culture of accountability, then it starts with us.

We need to model the behaviours that we want to see in our organization.

If we want our team to take ownership, then we have to be seen to take ownership.

When we make commitments we have to be seen to meet those commitments.

Altruism is another important part of professionalism.

Altruism is the unselfish concern for other people; doing things simply out of a desire to help, not because we feel obligated to out of duty, loyalty, or religious reasons.

This requires us to honour patients' wishes.

Excellent communication with patients and respect them.

Cultural needs should be considered.

Physicians may require disclosing errors to the patients and the hospital, despite any personal embarrassment, but at present, this is grossly under reported.

\section{Honour and Integrity:}

Physicians are expected to demonstrate the highest standards of behaviour. Physicians are faced with choices that may lead them astray on a daily basis. Financial gain is one of a multitude of potentially tempting opportunities to physicians. Integrity also means obeying laws and complying with regulations. 
In order to make an effort to derive the concepts in professional excellencein sexual health and HIV care, I believe we had a fruitful session on safeguarding children and young adults this morning during our pre-congress sessions. It was encouraging to see that we are not alone. We were able to join hands with college of paediatricians, UNICEF, college of forensic pathologists and college of psychiatrists. Furthermore, I would like to emphasis the fact that we have made every effort to create an environment to facilitate and provide opportunities to our members to gain exposure and bring-in best practices in excellence to our profession. In this direction, we have organized monthly lunch time $\mathrm{CPD}$ meetings on clinical governance, medicolegal aspects and other relevant topics. We initiated a monthly "virtual HIV clinic" to discuss and put our knowledge and experience together on management of difficult cases. We were able to get certified as an organization awarding CPD points to resource and participants of our educational events. A comprehensive guide on "management of sexual health problems" is in process.

Ladies and gentlemen, we as professionals have a moral obligation to work towards in achieving professional excellence in sexual health and HIV care.

Thank you.

Dr D. O. C. de Alwis ; President - SLCoSHH 